\title{
Solitary fibrous tumor of the greater omentum in an inguinal hernia sac
}

\author{
Keiichiro Uemura · Tokumasa Hayashi • \\ Kei Matsuoka
}

Received: 7 September 2011 / Accepted: 20 December 2011/Published online: 20 January 2012

(C) The Japan Society of Clinical Oncology 2012

\begin{abstract}
A 40-year-old man visited our hospital in December 2008 complaining of a painless mass in the left inguinoscrotal area. The surface of the mass, expected to be a tumor, was smooth and mobile. Ultrasonography showed the tumor was separated from the same-side testis, and extended to the spermatic cord. Computed tomography (CT) revealed that the heterogeneous tumor extended to the spermatic cord, and that the left inguinal canal was dilated. We diagnosed him with a left spermatic cord tumor, and left ascensus orchiectomy was performed on February 5th, 2009. With regard to the operative findings, the tumor was located in the inguinal hernia sac and extended to the greater omentum, and was separated from the spermatic cord. Immunohistochemical examination showed positive reactivity for CD99 and vimentin; however, desmin, S100, and bcl-2 were negative. CD34 was positive only in a few tumor cells around the blood vessel. The final diagnosis was solitary fibrous tumor (SFT), although it was not a representative type, originating from the greater omentum, existing in an inguinal hernia sac. This is the first such case to be reported anywhere in the world. So, we describe this rare case.
\end{abstract}

Keywords Solitary fibrous tumor - Inguinal hernia sac . Greater omentum

\section{Introduction}

Solitary fibrous tumor (SFT) is a rare tumor of submesothelial origin. Klemperer and Robin first described in 1931

K. Uemura $(\bowtie) \cdot$ T. Hayashi $\cdot$ K. Matsuoka

Department of Urology, Kurume University School of Medicine,

Asahimachi 67, Kurume, Fukuoka, Japan

e-mail: uemura_keiichirou@kurume-u.ac.jp how SFT originated in the pleural cavity. Recently, SFT has been incidentally found in other organs. Histologically, a proliferation of spindle cells of hemangioma-like and fibrous appearance are observed for SFT, and immunohistochemical findings are available for diagnosis. In most cases, SFT is associated with a favorable prognosis; sometimes, however, prognosis is poor, so long-term follow-up should be performed. We encountered a very rare patient with SFT in an inguinal hernia sac, in which the primary organ was the greater omentum.

\section{Case report}

In December 2008, a 40-year-old Japanese man visited our hospital because of a swelling in the left scrotum. Upon palpation, the tumor was located between the left testis and the inguinal region on the same-side, and the surface was smooth. The tumor was slightly movable. Ultrasonography showed that the tumor had a nearly uniform internal structure, and was separated from the testis. CT showed that the tumor extended to the spermatic cord (Fig. 1a), and that the left inguinal canal was dilated, because of protrusion by the greater omentum (Fig. 1b). Laboratory findings showed that testicular tumor markers (lactate dehydrogenase, human chronic gonadotropin, and $\alpha$-fetoproteins) were normal. We diagnosed him with a left spermatic cord tumor complicated by left inguinal hernia. We decided to perform a left ascensus orchiectomy and radical operation for inguinal hernia in February 2009.

In the operation, the tumor was found to be in the hernia sac, separate from the left spermatic cord (Fig. 2a). The tumor extended to the greater omentum (Fig. 2b), and the primary organ of the tumor seemed to be the greater omentum within the inguinal hernia sac. 

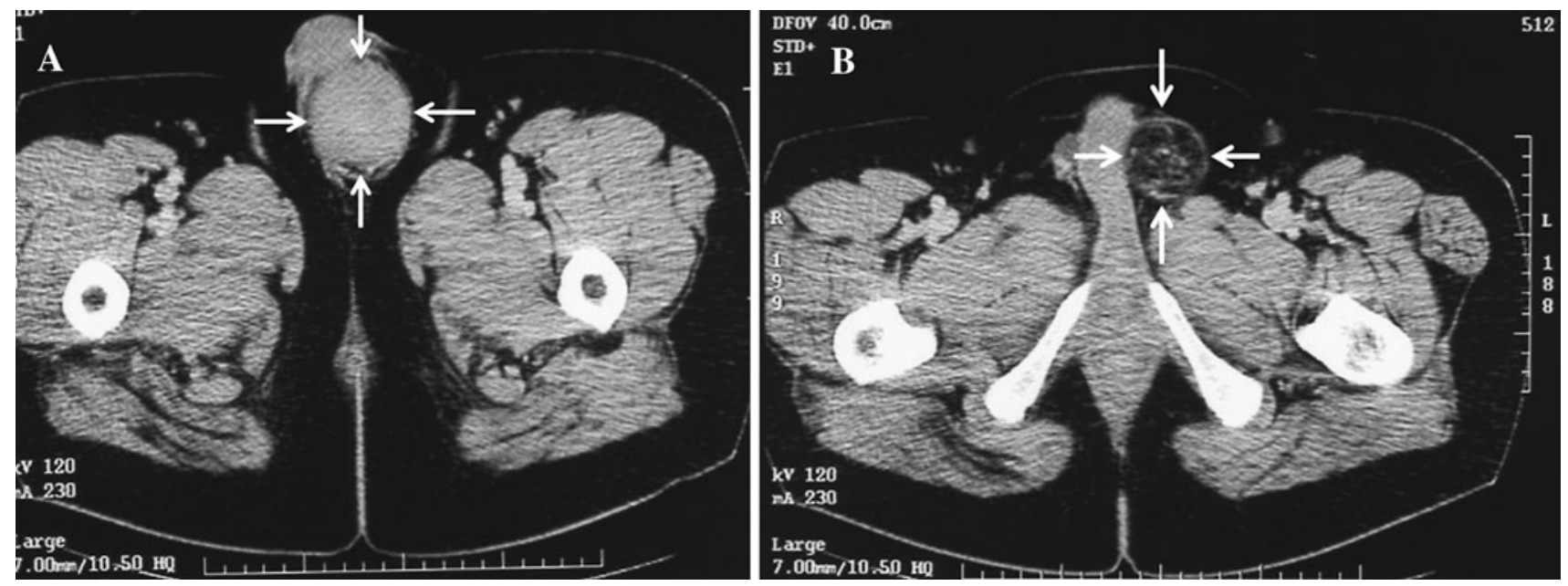

Fig. 1 Computed tomography (CT) findings. a The tumor is continuous with the spermatic cord. b The left inguinal canal is dilated, and there seems to be an inguinal hernia

Fig. 2 Operative findings. a The tumor was separate from the left spermatic cord, and located in the hernia sac. b The open hernia sac shows the tumor extending to the greater omentum
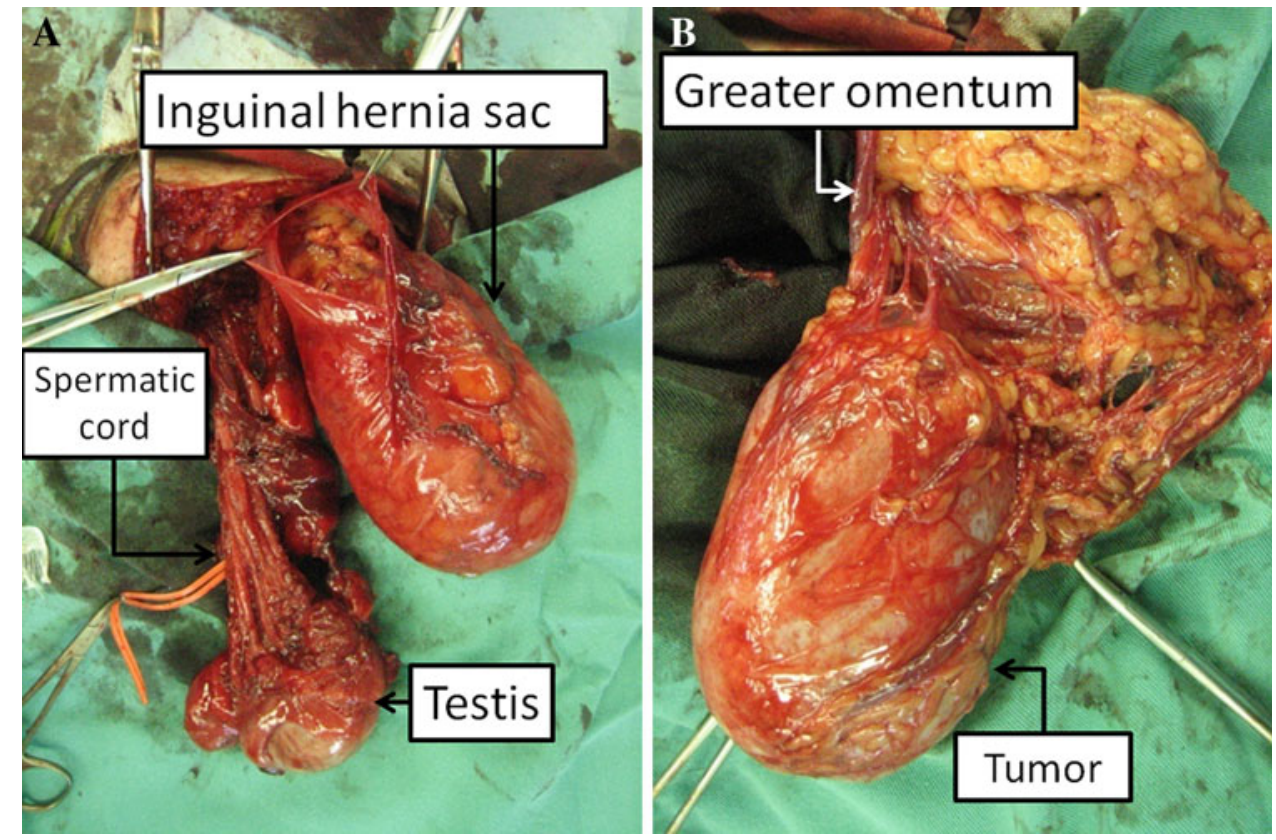

Pathology findings revealed a proliferation of spindle cells of hemangioma-like and fibrous appearance. No atypical cells were observed (Fig. 3a). With regard to immunohistochemical findings, a few tumor cells were positive for CD34 around the blood vessel (Fig. 3b), positive for CD99 (Fig. 3c), and negative for Desmin, S100, SMA, and bcl-2 (Fig. 3d).

On the basis of these findings, we made a final diagnosis of SFT; however, the tumor had a few CD34-positive cells. The patient recovered uneventfully, and there had been no recurrence at 2 years after the operation.

\section{Discussion}

Solitary fibrous tumor is a rare mesenchymal neoplasm. SFTs seldom originate from the omentum, and are usually pleura-based lesions. However, since the 1990s, reports of primary organs other than the pleura have increased. Nevertheless, the pleura tends to be the primary organ, at a ratio of 11 to 5 [1]. Various other primary organs have been reported, including the meninx, orbit, upper airway, salivary gland, thyroid gland, peritoneum, liver, retroperitoneum, pelvic cavity, adrenal gland, and kidney [2-4]. 

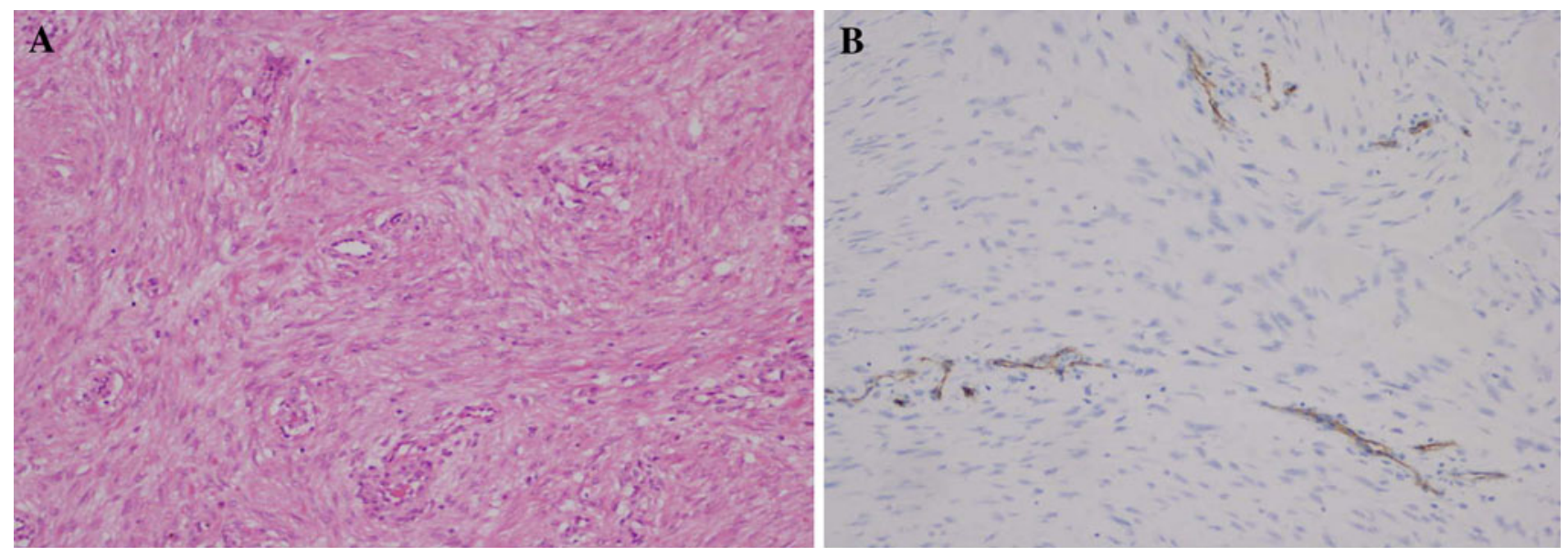

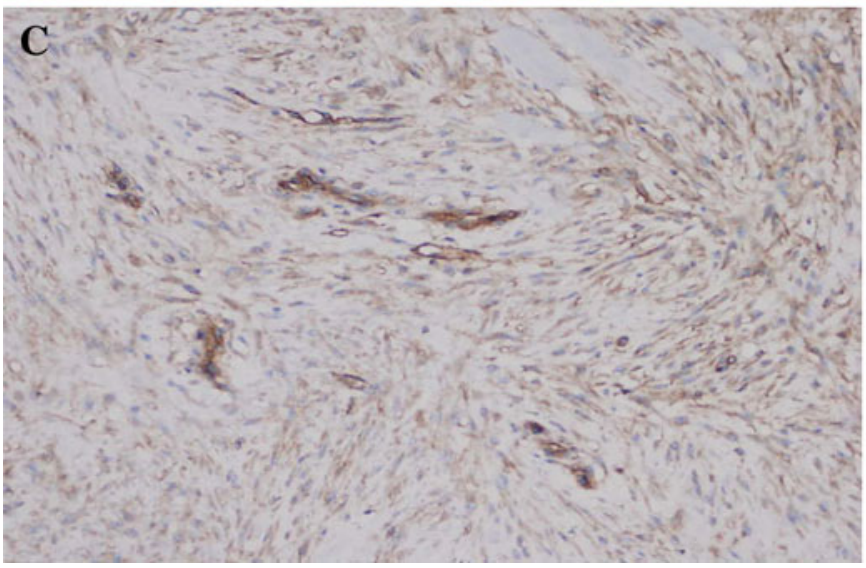

Fig. 3 Histological findings and immunohistochemical features of the tumor specimen. a The tumor consists of a proliferation of spindle cells with hemangiopericytoma-like appearance; no atypical cells are

There seems to be two mechanisms for the tumor, originating from the greater omentum, to exist in the inguinal hernia sac. In one, at the start, the tumor existed in the abdominal cavity. Then, at some point, it extended into the inguinal hernia sac. In the other, the tumor originated from the greater omentum, which existed in the inguinal hernia sac from the beginning.

No differences in clinical progress or pathological findings have been reported between SFT on the pleura and on other organs $[1,4,5]$.

Solitary fibrous tumor originating from the greater omentum is very rare, and only 6 cases have been reported (Table 1) [6]. This is the first time SFT from the greater omentum in a hernia sac has been described anywhere in the world. Six cases with greater omentum SFT were reviewed. There were 4 male and 2 female patients aged 24-77 years (mean \pm SE $49.0 \pm 8.0$ years), with tumor sizes from 32 to $230 \mathrm{~mm}$ (mean \pm SE $118.2 \pm 27.0 \mathrm{~mm}$ ).

Clinical symptoms of SFT not originating in the pleura were hypoglycemia and local symptoms of the tumor [7]. The local symptom in our case was left scrotum swelling,

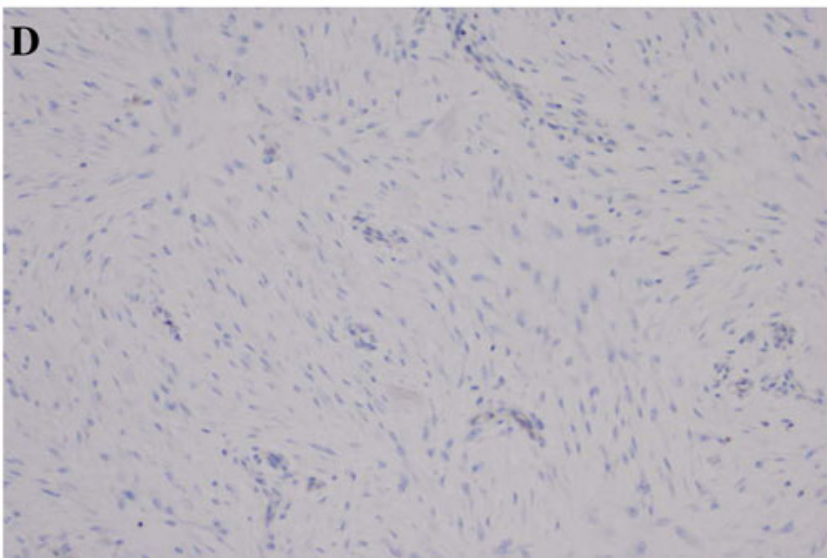

observed. $\mathrm{H} \& \mathrm{E} \times 100$. b CD34 is positive in some tumor cells around the blood vessel. c CD99 is positive in tumor cells. Immunostain $\times 100$. $\mathbf{d}$ bcl-2 is negative in tumor cells. Immunostain $\times 100$

Table 1 SFT of the greater omentum reported in the world

\begin{tabular}{llccll}
\hline Case & Sex & Age & $\begin{array}{c}\text { Major } \\
\text { axis } \\
\text { length } \\
\text { (mm) }\end{array}$ & Treatment & Recurrence \\
\hline Partriti et al. & M & 24 & 32 & Resection & No (2 years) \\
Takase et al. & F & 63 & 115 & Resection & Not mentioned \\
Kuroki et al. & F & 77 & 140 & Resection & Not mentioned \\
Yamano et al. & M & 55 & 230 & Resection & Not mentioned \\
Yoshida et al. & M & 35 & 82 & Resection & Not mentioned \\
Present case & M & 40 & 110 & Resection & No (8 months) \\
\hline
\end{tabular}

with no hypoglycemia. Briselli et al. [8] have reported that hypoglycemia occurred in approximately $4 \%$ in pleural SFT, and there has been no report of the symptom caused by greater omentum SFT. Hypoglycemia is a paraneoplastic syndrome of SFT. The tumor reportedly develops as a result of the production of a high-molecular-weight form of insulin-like growth factor-II (IGF-II). The highmolecular-weight form of IGF-II leads to the symptoms of 
hypoglycemia by two mechanisms. In one, IGF-II binds to the IGF-I receptor. In consequence, secretion of the growth hormone is suppressed at the pituitary gland, and the serum level of IGF-I is reduced. In the other, IGF-II is able to revitalize insulin receptors of the pancreas, inducing an increase in peripheral glucose and suppression of hepatic gluconeogenesis [9].

A characteristic pathological finding of SFT is an increase in the number of hemangio-pericytoma-like spindle cells with a fibrous appearance. With regard to immunohistochemical findings, CD34, bcl-2, and CD99 have been found to be helpful for diagnosis of SFT [7, 10]. In our case, the tumor cells were negative for bcl-2, positive for CD99, and a few were positive for CD34 around the blood vessel. Therefore, immunohistochemically, our case is not a typical SFT because many CD34-negative cells were present in the tumor.

Solitary fibrous tumor is usually a benign tumor, but $10-15 \%$ of all cases have malignant character [10]. The malignancy ratio of SFT originating outside the pleura is reported to be $9-18 \%[1,4,5,11]$. Prognostic factors of SFT are a tumor diameter over $100 \mathrm{~mm}$ [5], characteristic pathological findings, and immunohistochemical findings. Pathological criteria of malignancy are high mitotic activity (more than 4 mitoses per 10 high-power fields), high cellularity, pleomorphism, and necrosis $[1,5,12]$. Immunohistochemically, negative CD34 and positive p53 are reported as characteristic of malignant SFT [11].

In this case, the tumor diameter was over $100 \mathrm{~mm}$, and it was hard to observe the CD34 immunopositive tumor cells within the parenchyma. Consequently, careful longterm follow-up was mandatory.

Treatment for SFT is resection of the tumor with a sufficient surgical margin $[5,13]$. The incidence of recurrence and metastasis of SFT is $0-19 \%$ over $1-14$ years [2, 14, 15]. Approximately $80 \%$ of recurrent cases are malignant SFT, and recurrent benign SFT is rarely reported [2]. Therefore, SFT has malignant potential. Treatment for recurrent cases is usually surgical resection, but a few reports have indicated that radiotherapy, and chemotherapy with ifosfamide and adriamycin may be effective [5, 13]. However, these treatments are not yet established.

More reports of SFT are required before a standard treatment can be established.

Conflict of interest No author has any conflict of interest.

\section{References}

1. Vallat-Decouvelaere AV, Dry SM, Fletcher CDM (1998) Atypical and malignant solitary fibrous tumors in extrathoracic locations. Am J Surg Pathol 22:1501-1511

2. van de Rijn M, Lombard CM, Rouse RV (1994) Expression of CD34 by solitary fibrous tumors of the pleura, mediastinum, and lung. Am J Surg Pathol 18:814-820

3. Chilosi M, Facchetti F, Dei Tos AP et al (1997) bcl-2 expression in pleural and extrapleural solitary fibrous tumors. J Pathol 181:362-367

4. Hasegawa T, Matsuno Y, Shimoda T, Hasegawa F, Sano T, Hirohashi $S$ (1999) Extrathoracic solitary fibrous tumors: their histological variability and potentially aggressive behavior. Hum Pathol 30:1464-1473

5. Goli JS, Antonescu CR, Hanju C, Ferrone CR, Hussain M, Lewis JJ et al (2002) Clinicopathologic correlates of solitary fibrous tumors. Cancer 94:1057-1068

6. Patriti A, Rondelli F, Gulla N, Donini A (2006) Laparoscopic treatment of a solitary fibrous tumor of the greater omentum presenting as spontaneous haemoperitoneum. Ann Ital Chir 77:351-353

7. Weiss SW, Goldblum JR (2001) Solitary fibrous tumor. In: Eizinger and Weiss's soft tissue tumors, 4th edn. Mosby, St Louis, pp 1021-1035

8. Briselli M, Mark EJ, Dickersin GR (1981) Solitary fibrous tumors of the pleura: eight new cases and review of 360 cases in the literature. Cancer 47:2678-2689

9. Yamakawa-Yokota F, Ozaki N, Okajima A, Nishio H, Nagasaka T, Oiso Y (2010) Retroperitoneal solitary fibrous tumor-induced hypoglycemia associated with high molecular weight insulin-like growth factor II. Clin Med Res 8:159-162

10. Guillou L, Fletcher JA, Fletcher CDM, Mandahl N (2002) Extrapleural solitary fibrous tumor and haemangiopericytoma. In: Fletcher CDM, Unni KK, Mertens F (eds) World Health Organization classification of tumors. Pathology and genetics, tumors of soft tissue and bone. IARC Press, Lyon, pp 86-90

11. Yokoi T, Tsuzuki T, Yatabe Y, Kurumaya H, Koshikawa T, Kakubo K et al (1998) Solitary fibrous tumor: significance of p53 and CD34 immunoreactivity in its malignant transformation. Histopathology 32:423-432

12. England DM, Hochholzer L, McCrathy MJ (1989) Localized benign and malignant fibrous tumors of the pleura. Am J Surg Pathol 13:640-658

13. de Perrot M, Fischer S, Brundler MA et al (2002) Solitary fibrous tumor of the pleura. Ann Thorac Surg. 74:285-293

14. Renshaw AA, Pinkus GS, Corson JM (1994) CD34 and AEI/ AE3; diagnostic discriminants in the distinction of solitary fibrous tumor of the pleura from sarcomatoid mesothelioma. Appl Immunohistochem 2:94-102

15. Tsuchiya K, Fukuse T, Satoda N, Ogawa H, Saiga T (2006) A case of solitary fibrous tumor of the retroperitoneum, metastatic to the lung and the parotid grand after an 11-year disease free interval. Jpn J Lung Cancer 46:369-374 